\title{
Structural properties of the active layer of discotic hexabenzocoronene/perylene diimide bulk hetero junction photovoltaic devices: The role of alkyl side chain length
}

\author{
M. Al-Hussein ${ }^{\text {a, }}$, H.C. Hesse ${ }^{\text {b }}$, J. Weickert ${ }^{\text {b }}$, L. Dössel ${ }^{\text {c }, ~ X . ~ F e n g ~}{ }^{c}$, K. Müllen ${ }^{\text {c }, ~ L . ~ S c h m i d t-M e n d e ~}{ }^{\text {b }}$ \\ a Department of Physics, University of Jordan, Amman 11942, Jordan \\ b Ludwig-Maximilians-University Munich, Department of Physics and Center for NanoScience(CeNS), Amalienstr.54, 80799 Munich, Germany \\ ' Max Planck Institute for Polymer Research, Ackermannweg 10, 55128 Mainz, Germany
}

Keywords:

Organic solar cells

Bulk-heterojunction

Discotic liquid crystal

Active layer morphology

Scanning electron microscopy

$\mathrm{X}$-ray diffraction

\begin{abstract}
A B S T R A C T
We investigate thin blend films of phenyl-substituted hexa-peri-hexabenzocoronenes (HBC) with various alkyl side chain lengths $\left(\left(\mathrm{CH}_{2}\right) \mathrm{n}, \mathrm{n}=6,8,12\right.$ and 16$)$ /perylenediimide (PDI). These blends constitute the active layers in bulk-hetero junction organic solar cells we studied recently [1]. Their structural properties are studied by both scanning electron microscopy and X-ray diffraction measurements. The results support the evidence for the formation of HBC donor-PDI acceptor complexes in all blends regardless of the side chain length of the $\mathrm{HBC}$ molecule. These complexes are packed into a layered structure parallel to the substrate for short side chain HBC molecules $(n=6$ and 8 ). The layered structure is disrupted by increasing the side chain length of the HBC molecule and eventually a disordered structure is formed for long side chains $(n>12)$. We attribute this behavior to the size difference between the aromatic parts of the HBC and PDI molecules. For short side chains, the size difference results in a room for the side chains of the two molecules to fill in the space around the aromatic cores. For long side chains $(n>12)$, the empty space will not be enough to accommodate this increase, leading to the disruption of the layered structure and a rather disordered structure is formed. Our results highlight the importance of the donor-acceptor interaction in a bulk heterojunction active layer as well as the geometry of the two molecules and their role in determining the structure of the active layer and thus their photovoltaic performance.
\end{abstract}

\section{Introduction}

Bulk-heterojunction (BHJ) organic solar cells have recently attracted considerable attention owing to their potential low cost fabrication with currently maximum efficiency exceeds $7 \%$ [2-6]. The solution-processed active layer in these cells is formed by blending two self-assembling organic semiconductors: a hole-transporting donor (D), and an electron-transporting acceptor (A). Ideally, the two semiconductors have to vertically segregate while maintaining a large interfacial surface area. This ensures an unperturbed transport of positive and negative charge carriers to their respective electrodes and effective charge separation needed to achieve highest power conversion efficiency. Therefore, the structure and nanoscale morphology of the active layer are of major importance for the performance of a $\mathrm{BH}$ organic solar cell. Another prerequisite of the semiconductors is high charge-carrier mobility. A promising candidate for such organic semiconductors is offered by conjugated discotic liquid crystals [7-10]. Their flat, disk-shaped aromatic, cores with strong $\pi-\pi$ interactions promote their self-assembly into columnar stacks that allow electric

\footnotetext{
* Corresponding author.

E-mail address: m.alhussein@ju.edu.jo (M. Al-Hussein).
}

charges to migrate because of the overlap of the extended $\pi$-orbitals of their cores. Of particular importance of this family are the hexa-perihexabenzocoronenes (HBCs) [11-15]. They have shown unusually high charge-carrier mobilities along the $\pi$-stacked columns [16-18] These electron-rich mesogens are p-type semiconductors, [19] thus promoting efficient hole transport. Chemical modification of these molecules by side-chain substituents allows tailoring their physicochemical properties [20-23]. Moreover, the side chains, typically alkyl render the molecules soluble and thereby enable their processing in solution using common methods such as spin-coating and solutioncasting. However, the length of the side chains affects the field-effect mobility and more importantly the self-assembly behavior of the active layer, and thus the solar cell performance.

In our previous study, BHJ solar cells with an inverted architecture have been demonstrated using different $\mathrm{HBC}$ donor molecules blended with rod-shaped perylenediimide (PDI) as the acceptor material [1]. We investigated the effect of varying the alkyl side chain length, $n=6$, 8,12 and 16 carbon atoms on the cell performance. The results showed that $\mathrm{BHJ}$ solar cells made of $\mathrm{HBC}$ derivatives with short alkyl side chains ( $n=6,8$ and to some extent 12) showed better performance than those made of HBC derivatives with long alkyl side chains $(n=16)$. A summary of the results obtained in our previous study is presented in Table 1. 
Table 1

Summary of the characteristics of the different HBC:PDI solar cells from ref. [1].

\begin{tabular}{llll}
\hline Blend & $I_{S C}\left(\mathrm{~mA} / \mathrm{cm}^{2}\right)$ & $\mathrm{V}_{\mathrm{OC}}(\mathrm{V})$ & PCE $(\%)$ \\
\hline HBC-6:PDI & $-1.035 \pm 0.042$ & $0.643 \pm 0.023$ & $0.22 \pm 0.02$ \\
HBC-8:PDI & $-1.065 \pm 0.035$ & $0.665 \pm 0.026$ & $0.24 \pm 0.02$ \\
HBC-12:PDI & $-0.815 \pm 0.050$ & $0.690 \pm 0.017$ & $0.22 \pm 0.02$ \\
HBC-16:PDI & $-0.340 \pm 0.027$ & $0.593 \pm 0.065$ & $0.08 \pm 0.02$ \\
\hline
\end{tabular}

Structure and morphology of the active layer in the different investigated cells are central to understand such differences in their photovoltaic performance.

The present study aims to elucidate the influence of the alkyl side chain length of the $\mathrm{HBC}$ donor molecule on the organizational principles and intermolecular packing in the active layers made of HBC donor: PDI acceptor blend. This will help to understand the difference in the solar cell performance reported in our previous work. We systematically characterize the inner structure and surface morphology of thin films of pure layers of PDI molecules and HBC molecules with varying alkyl side chain length as well as their blends with perylene diimide by X-ray diffraction and scanning electron microscopy, respectively. The results of the X-ray experiments support the evidence for the formation of donor-acceptor complexes in all blends regardless of the side chain length of the HBC molecule. However, for short side chain HBC molecules $(n=6,8,12)$, these complexes are packed into layers parallel to the substrate, whereas a disordered structure is formed for the blend of HBC with $n=16$.

\section{Experimental details}

\subsection{Materials}

All materials used in this study are summarized in Fig. 1. Synthesis of the phenyl-substituted hexaphenyl-substituted HBCs with different alkyl chain lengths (HBC-6, HBC-8, HBC-12 and HBC-16) was accomplished using synthesis procedures reported elsewhere [1,24]. After synthesis, all compounds were purified by repetitive precipitation and silica column chromatography to remove metal ions and impurities. Perylene tetracarboxdiimide (PDI) was bought from Sensient technologies and used as received.

The thermotropic properties of the synthesized components were analyzed using differential scanning calorimetry (DSC) and 2D powder $\mathrm{X}$-ray diffraction. All HBC compounds show a liquid crystalline phase with at least one accessible phase transition. The thermotropic behavior of all studied compounds is summarized in Table 2 .

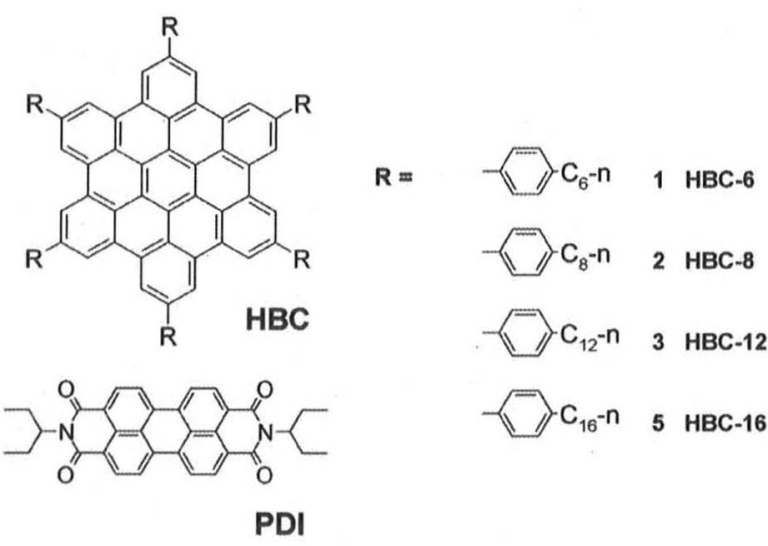

Fig. 1. Chemical structure of organic materials used in this study. HBC-x: phenylsubstituted hexa-peri-hexabenzocoronene $(\mathrm{HBC})$ with different alkyl side chains $(\mathrm{x})$; perylenediimide (PDI).
Table 2

Thermotropic properties of the different derivatives obtained using differential scanning calorimetry (DSC) and 2D powder X-ray diffraction.

\begin{tabular}{llll}
\hline Derivative & Phase transitions & Core-core spacing & Column-column spacing \\
\hline PDI & $68^{\circ} \mathrm{C}, \mathrm{Cr}-\mathrm{Cr}$ & $0.35 \mathrm{~nm}$ & $1.61 \mathrm{~nm}$ \\
HBC-6 & $161^{\circ} \mathrm{C}, \mathrm{LC}-\mathrm{LC}$ & $0.35 \mathrm{~nm}$ & $2.76 \mathrm{~nm}$ \\
$\mathrm{HBC}-8$ & $153^{\circ} \mathrm{C}$, LC$-\mathrm{LC}$ & $0.35 \mathrm{~nm}$ & $2.97 \mathrm{~nm}$ \\
$\mathrm{HBC}-12$ & $-22^{\circ} \mathrm{C}, 62^{\circ} \mathrm{C}$, LC-LC & $0.35 \mathrm{~nm}$ & $3.42 \mathrm{~nm}$ \\
$\mathrm{HBC}-16$ & $9^{\circ} \mathrm{C}, \mathrm{LC}-\mathrm{LC}$ & $0.35 \mathrm{~nm}$ & $3.70 \mathrm{~nm}$ \\
\hline
\end{tabular}

Phase assignment: $\mathrm{Cr}=$ crystalline phase; $\mathrm{LC}=$ liquid crystalline phase; $\mathrm{I}=$ isotropic phase.

The active layers studied in this work were prepared using the same procedure that we used to fabricate the photovoltaic devices in our previous study [1]. In brief, indium tin oxide (ITO) substrates were cleaned by ultrasonication in acetone and isopropyl alcohol bath followed by a $7 \mathrm{~min}_{2}$ plasma (Diener Femto) cleaning. About $150 \mathrm{~nm}$ of $\mathrm{TiO}_{2}$ were deposited onto the cleaned ITO substrates by spray pyrolysis at $450^{\circ} \mathrm{C}$ using a diisopropoxytitanium bis(acetylacetonate) precursor solution. It is worth mentioning here that the use of the $\mathrm{TiO}_{2}$ layer in our devices was to ensure an inverted architecture. Organic solar cell using a non-corrosive Au metal as the back electrode and transparent $\mathrm{TiO}_{2}$ as the front electrode showed high durability in an ambient atmosphere [25-29]. HBC and PDI molecules were dissolved in chloroform at a concentration of $15 \mathrm{mg} / \mathrm{ml}$ and mixed at $40: 60 \mathrm{wt} . \%$ HBC:PDI. The blend solution was stirred for $48 \mathrm{~h}$ and filtered $(45 \mu \mathrm{m}$ pore) immediately before use. The organic layers (pure or blend) were deposited by spin coating $(2000 \mathrm{rpm})$, resulting in an average film thickness of about $120 \mathrm{~nm}$. The films were annealed for $1 \mathrm{~h}$ at $120^{\circ} \mathrm{C}$ at ambient atmosphere prior to testing.

\subsection{Scanning Electron Microscopy (SEM)}

The surface morphology of the blend films was analyzed using a LEO FE-REM DSM 982 scanning electron microscope. The imaging was performed at $8 \mathrm{kV}$ with a working distance of $10-20 \mathrm{~mm}$. Surfaces were scanned with no further modification (no $\mathrm{Au} / \mathrm{Cr}$ sputtering) in order to visualize the morphology of the organic layer only.

\subsection{X-ray diffraction}

X-Ray diffraction measurements were performed using a fourcircle diffractometer. The radiation used was a Ge (111) monochromatized $\operatorname{MoK} \alpha$ beam $(\lambda=0.71 \AA)$ from a sealed-tube generator $(900 \mathrm{~W})$. The $\mathrm{X}$-ray scattering vector $q$ is defined as the difference between the scattered and the incident X-ray wave vectors. The diffraction curves were recorded by a scintillation detector. Exposure times were varied between 1 and $24 \mathrm{~h}$.

\section{Results and discussion}

\subsection{Surface morphology}

Fig. 2 shows SEM images of the surface topology of the blend materials. Flake-like domains are clearly visible for the HBC-6:PDI and HBC-8:PDI blends. The domains become smaller for the HBC-12:PDI blend, whereas the HBC16-PDI blend shows a rather fine texture. Pure $\mathrm{HBC}$ films exhibit rather smooth and almost featureless surface structures [11]. The larger domains for the short side chain $\mathrm{HBC}$ blends are indicative of a better packed material. It is known that, increased crystallite size allows for better percolation pathways towards the contacts [30]. In contrast, disordered regions lead to lower charge carrier mobility and thus lower currents. To elucidate the inner structures of the flake-like domains, we analyzed the same blend films using X-ray diffraction. 
A

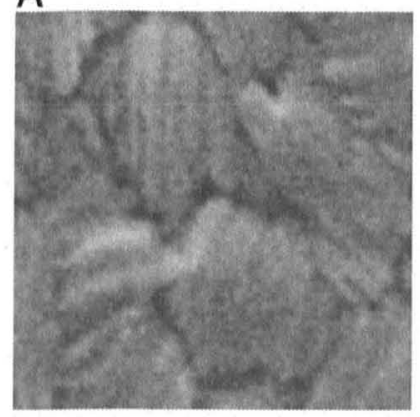

C

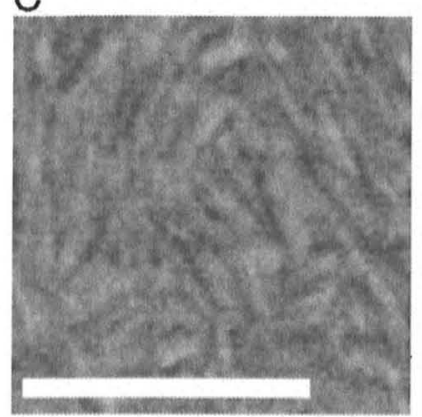

B

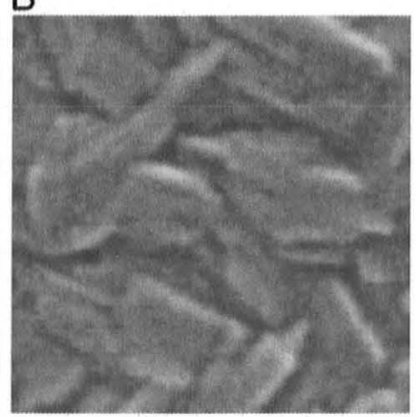

D

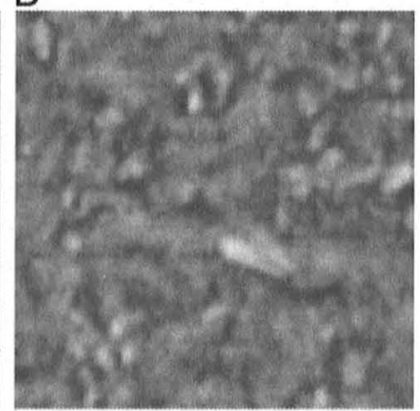

Fig. 2. SEM images of the surfaces of the different HBC-PDI blends: A) HBC-6:PDI, B) HBC-8:PDI, C) HBC-12:PDI, D) HBC-16:PDI. The bar size is $500 \mathrm{~nm}$.

\subsection{Inner film structure}

\subsubsection{Substrate effect}

Direct information about the microstructure of the films can be obtained by X-ray diffraction measurements [31]. As a preliminary step, we investigated the X-ray patterns of both clean ITO covered glass, and $\mathrm{TiO}_{2} / \mathrm{ITO}$ covered glass substrates using Bragg-Brentano geometry (Fig. 3). As can be seen, both substrates exhibit diffraction peaks in the range of $1.5-2.0 \AA^{-1}$, superimposed on a broad halo of the glass. The peak at $q_{1}$ is due to the ITO indicating its crystalline nature [32]. Meanwhile, the peak at $q_{2}$ is due to the $\mathrm{TiO}_{2}$ layer. Comparing with the literature data, it can be concluded that $q_{2}$ peak is the (100) reflection of the anatase crystalline structure of the $\mathrm{TiO}_{2}$ layer [33]. These two peaks, $q_{1}$ and $q_{2}$, were also seen in all X-ray diffraction

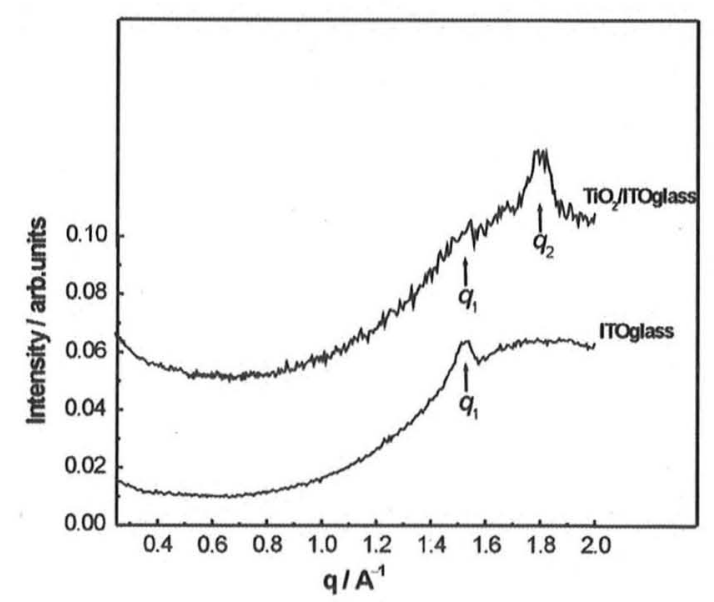

Fig. 3. X-ray diffraction curves obtained using Bragg-Brentano (B-B) geometry for clean ITO covered glass, and $\mathrm{TiO}_{2} /$ ITO covered glass substrates. The arrows indicate diffraction peaks.

patterns obtained for films of either pure HBC and PDI derivatives or their blends on $\mathrm{TiO}_{2} / \mathrm{ITO}$ covered glass substrates using the BraggBrentano geometry as can be seen in Fig. 4 (lower curve), as an example. This has limiting consequences on analyzing the large $q$ range of the X-ray diffraction patterns of either pure derivatives or their blends. A possible $\pi$-stacking of the conjugated cores normal to the substrate (face-on arrangement) and ordering of the alkyl side chains are expected to show reflections in this $q$ range, i.e., coinciding with $q_{1}$ and $q_{2}$ peaks. Therefore, in order to ascertain any core-core stacking in a possible face-on arrangement in the active layer, the substrate effect ( $q_{1}$ and $q_{2}$ ) has to be eliminated or at least minimized. A common procedure, widely reported in the literature, used to minimize the substrate effect is to use grazing incidence diffraction geometry instead of the Bragg-Brentano one $[34,35]$. This is achieved by fixing the angle between the film surface and the incident beam, $\alpha_{\mathrm{i}}$, at a value close to the critical angle of the substrate and scanning the detector in the plane formed by the incident beam and the normal to the film surface. Fig. 4 (upper curve) shows diffraction curves obtained at $\alpha_{i}=0.18^{\circ}$ for the same blend sample used in Fig. 3. It is evident that even at such small incident angle, the substrate effect is not completely eliminated. This might be due to the large scattering depth obtained at large angles between the diffracted beam and the surface, $\alpha_{f}$, although $\alpha_{\mathrm{i}}$ is close to the critical angle. In fact, Dosch et al. have shown that the scanning of $\alpha_{\mathrm{f}}$ at constant incident angle varies the scattering depth and there is a symmetric influence of $\alpha_{\mathrm{i}}$ and $\alpha_{\mathrm{f}}$ upon the scattering depth [36]. Therefore, we concluded that using the $\mathrm{TiO}_{2} / \mathrm{ITO}$ covered glass substrates and our X-ray diffractometer, it would be hard to extract any reliable information concerning weak core-core stacking or alkyl side chains ordering oriented along the normal to the substrate in the active layer confidently. Nonetheless, no substrate effect is seen on the small $q$-range of the X-ray curve $\left(q<1.2 \AA^{-1}\right)$. On this basis, we will restrict ourselves to discuss the film microstructure using the small $q$ region of the curve only. This corresponds to ordering at large sizes corresponding to the arrangements of different phases in the active layer.

\subsubsection{Pure films}

We start by investigating the molecular order and packing behavior of each of the HBC derivatives as well as PDI films separately before we discuss the order and packing of their blends. Fig. 5 shows the X-ray diffraction patterns of pure HBC-6, HBC-8, HBC-12, HBC-16, and PDI films on $\mathrm{TiO}_{2} / \mathrm{ITO}$ covered glass substrates. The HBC- 6 and $\mathrm{HBC}-8$ films exhibit single diffraction peaks, whereas the HBC-12, HBC-16 and PDI films show narrower peaks at low $q$ with second order peaks at a relative

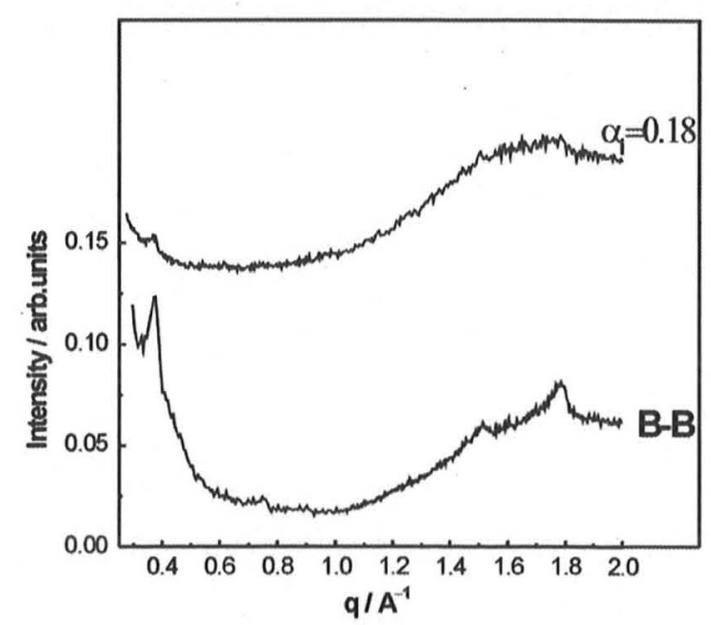

Fig. 4. X-ray diffraction curves of HBC-6:PDI blend obtained using Bragg-Brentano (B-B) geometry, and grazing-incidence geometry at $\alpha_{i}=0.18^{\circ}$. 

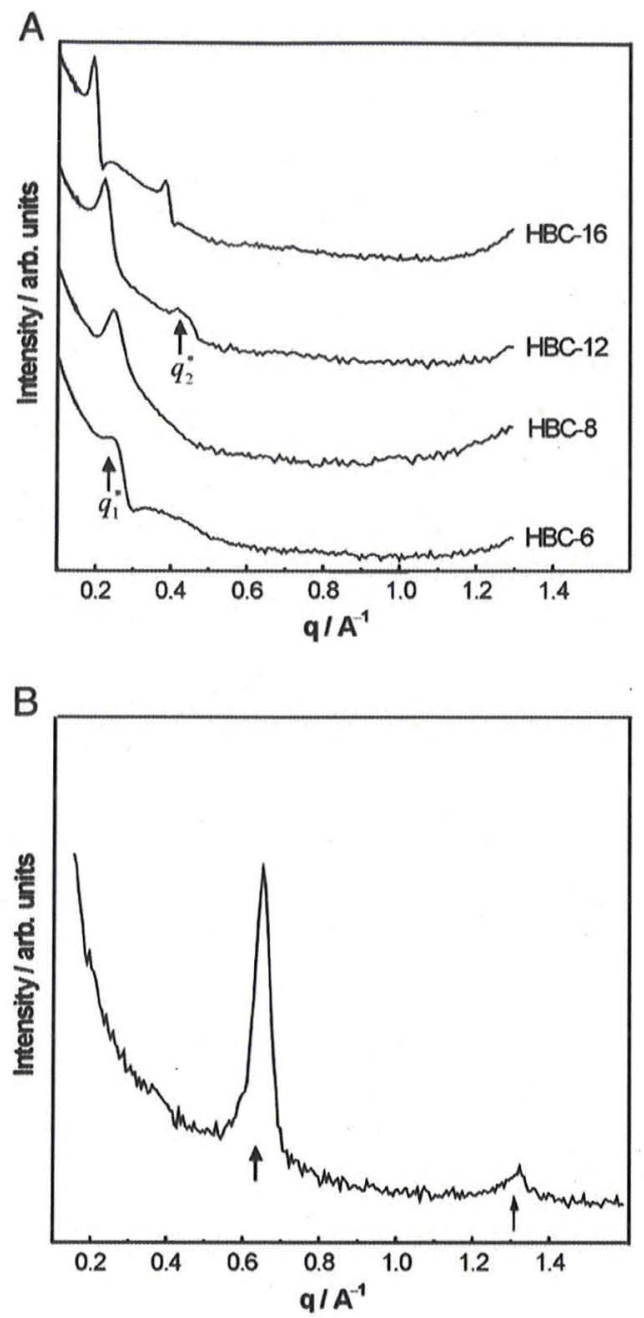

Fig. 5. X-ray diffraction curves obtained using Bragg-Brentano (B-B) geometry for (A) pure HBC derivatives (B) pure PDI. The arrows indicate diffraction peaks.

position ratio of $1: 2$. These observations indicate that microphase separation of the aliphatic and aromatic parts of both HBC and PDI molecules takes place and leads to a layered structure parallel to the substrate in each film. The appearance of a second order peak and the decrease in the full width at half maximum (FWHM) of the first order peak indicate sharp interfaces and a very regular repetition of these layers over a long distance of correlation length for the HBC-12, HBC-16 and PDI films. The corresponding interlayer spacing, $d$, can be calculated using Bragg's law, Table 3. As can be seen, the interlayer spacing is increasing while the FWHM is decreasing with increasing the side chain length of the $\mathrm{HBC}$ molecule. Consequently, we conclude that increasing the side length leads to better segregation as well as higher order and larger interlayer spacing of the $\mathrm{HBC}$ molecules. Both the incompatibility between the aliphatic and aromatic parts, and the $\pi-\pi$ interaction

Table 3

Bragg, calculated $d$-spacing and corresponding FWHM of the first-order peaks of Fig. 5.

\begin{tabular}{lcll}
\hline Derivative & $d$ (Bragg) & $d$ (calculated) & FWHM \\
\hline PDI & $9.6 \AA$ & - & $0.038 \AA^{-1}$ \\
HBC-6 & $25.0 \AA$ & $25.1 \AA$ & $0.051 \AA^{-1}$ \\
HBC-8 & $25.7 \AA$ & $25.6 \AA$ & $0.035 \AA^{-1}$ \\
HBC-12 & $28.6 \AA$ & $30.6 \AA$ & $0.022 \AA^{-1}$ \\
HBC-16 & $33.1 \AA$ & $35.7 \AA$ & $0.015 \AA^{-1}$ \\
\hline
\end{tabular}

between the aromatic conjugated cores drive the $\mathrm{HBC}$ and $\mathrm{PDI}$ molecules to form microphase-separated layers (columnar layers). Longer side chains lead to higher effective interaction parameter between the aliphatic side chains and the aromatic cores and thus increased microphase separation driving force resulting in sharper interfaces for the HBC derivatives.

To come up with structural models that describe the intermolecular ordering in the HBC and the PDI films, we need to be more quantitative. By considering the diameter of the aromatic core for each of the $\mathrm{HBC}$ derivatives, $16.8 \AA$, and the fully extended length of the alkyl side chain, the interlayer spacing, $d$, is calculated for each derivative assuming edge-on layered structure with interdigitated side chains, Fig. 6(A). The calculated values are listed in Table 3. As can be seen, the measured values are shorter than the calculated ones for longer side chain derivatives. This may be attributed to a slight tilt between the aromatic and aliphatic parts. As for the PDI film, the length of the aromatic part is $11.2 \AA$, while the width is $5.6 \AA$ only. The interlayer spacing derived from Fig. 5 is $9.6 \AA$. This is less than the full length of the aromatic part and therefore some tilt must be assumed in each layer Fig. 6 (B).

\subsubsection{Blend films}

In general, many factors affect the structure of the active layer: processing conditions, side-chain length, and material properties of the components such as their mutual miscibility, surface energies, and crystallinity $[37,39]$. Fig. 7 shows X-ray diffraction patterns for the different HBC-PDI blends obtained using Bragg-Brentano geometry. We emphasize here that the blends were prepared under the same conditions used in their corresponding solar cells, except the top metal layer deposition step, to ensure similar structure of the active layer. As can be seen, the curves of HBC-6:PDI and HBC-8:PDI blends show similar behavior whereby a relatively narrow peak, $\mathrm{q}_{1}{ }^{* *}$, with a weaker second order peak at a relative position of $1: 2$ is observed. The curve of
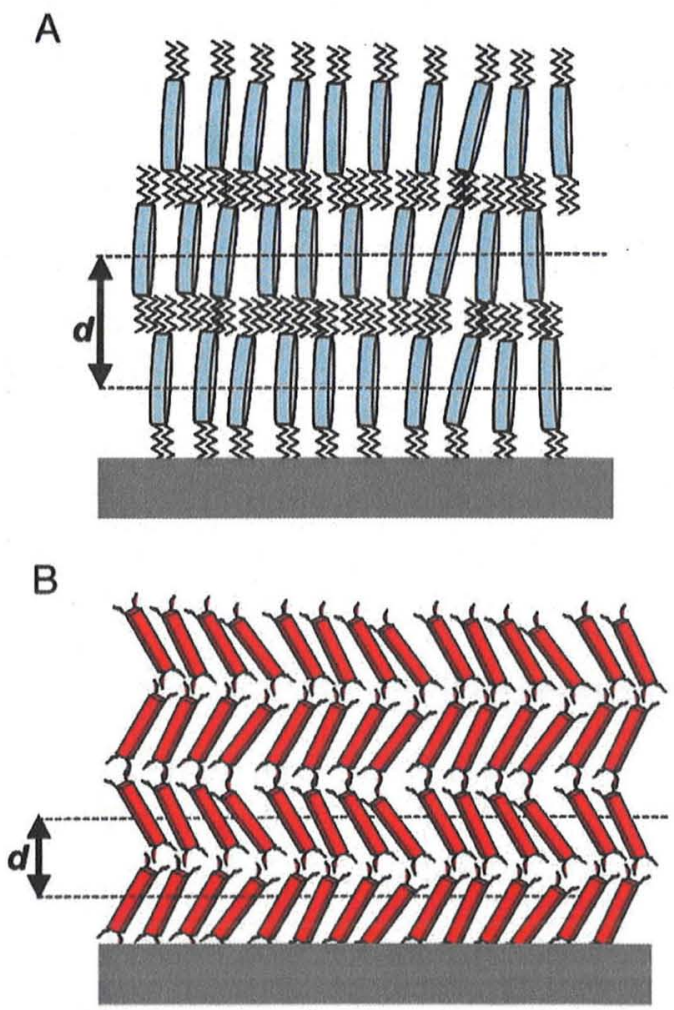

Fig. 6. Schematic drawing for a possible structural model of (A) pure HBC derivatives films, (B) pure PDI film. 


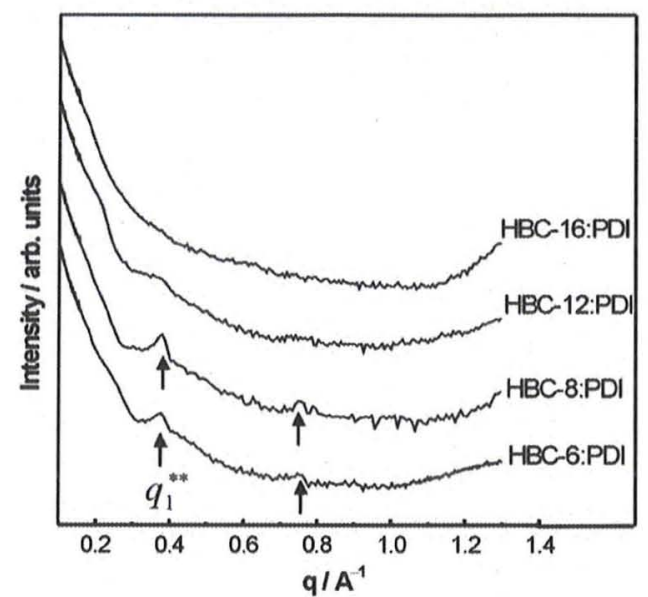

Fig. 7. X-ray diffraction curves obtained using Bragg-Brentano (B-B) geometry for the HBC:PDI blend films. The arrows indicate diffraction peaks.

the HBC-12:PDI blend exhibits a single broad weak peak, whereas the HBC16-PDI blend is almost featureless. These observations clearly indicate that a microphase separated layered structure parallel to the substrate is formed in the blends for the short side chain length $\mathrm{HBC}$ molecules (HBC-6 and $\mathrm{HBC}-8$ ). The narrow first-order peaks and the appearance of second-order peaks indicate better packing and larger domains in these blends in accordance with the SEM results (Fig. 2). Meanwhile, the weak single peak of the HBC-12:PDI blend and the almost featureless curve of the HBC-16:PDI blend indicate that the layered microstructure becomes more disordered with increasing the side chain length of the HBC molecules, and eventually a predominantly disordered structure is formed in the HBC-16:PDI blend.

Interestingly, the first-order peaks of the HBC-6:PDI, HBC-8:PDI and the single peak of the HBC-12:PDI all lie at almost the same $q$ value $\left(\sim 0.38 \AA^{-1}\right)$. Note that this value is different from the first-order peak position of either of the corresponding pure HBC or PDI layers for each of the three blends (Fig. 5). Therefore, it seems reasonable to conclude that the microphase separated layered structure in each blend is not simply templated by either of the HBC and PDI components. In contrast to the pure HBC layers, the almost constant $q$ value of the first order peak of the blends indicates a constant interlayer spacing. This is somewhat surprising as one would expect the interlayer spacing to increase with increasing the side chain length and in turn lower $q$ values would be observed for blends of $\mathrm{HBC}$ molecules with longer side chain. Evidently, the blends develop a layered structure that is not sensitive to the side chain length, at least up to $n=12$. The question arising now is how can we explain these observations in terms of a structural model? The miscibility (phase separation) of the diskshaped HBC and rod-shaped PDI molecules, alkyl side-chain packing, and $\pi$-stacking is the main driving force for the final morphology of the blend on the nanoscale. Kinetics often plays a role in the formation of film morphology of blends depending on solvent evaporation conditions. In contrast to blends of conjugated polymers which are generally not miscible at the molecular scale for entropic reasons, partial or even full miscibility commonly occurs for low molecular weight mesogens such as HBC and PDI despite their dissimilar shapes [37-39]. The fact that the diffraction pattern of each blend is different from either of its corresponding $\mathrm{HBC}$ and PDI components clearly suggests that no large-scale macrophase separation takes place between the HBC and PDI molecules (i.e., the blend is miscible). Such miscibility may be explained by the formation of donor-acceptor complexes as a result of the intermolecular interaction between donors and acceptors. Meanwhile, the strong $\pi-\pi$ interactions between the PDI cores prevent a random insertion of the PDI molecules between the layers (columns) of the HBC structure within the alkyl side chains, especially for such a relatively high amount of PDI (60 wt.\%).

The Bragg interlayer spacing, $d$, for the three layered blends is around $16.5 \AA$. Interestingly, this value comes very close to the diameter of the $\mathrm{HBC}$ molecules. On this basis, a possible model for the blend structure may be envisaged as shown in Fig. 8. The presence of donor-acceptor interaction stabilizes the intercalation of PDI (the acceptor) within layers of $\mathrm{HBC}$ disks resulting in stacking of the molecules parallel to the substrate. The size difference between the two aromatic parts in the HBC and PDI molecules $(\sim 6 \AA)$ results in a room for the side chains of the two molecules to fill in the space around the aromatic cores, particularly if the hard disks of $\mathrm{HBC}$ are not tilted. Apparently, as the side chain length of the HBC molecules increases from 6 to 8 carbons, the room will accommodate this increase resulting in an essentially constant interlayer spacing. At $n=12$, the side chains start to disrupt this layered structure. For side chain with $n>12$, the empty space produced by the size difference will not be enough to accommodate this increase. This leads to the disruption of the layered structure and instead a rather disordered structure is formed, as it is the case for the HBC-16:PDI blend. We recall here that the pure $\mathrm{HBC}-16$ film developed the most segregated layered structure (Fig. 5). However, blending the same molecule with PDI leads to almost disordered structure. This represents another support for the formation of donor-acceptor complexes in the blend. A layered structure normal to the substrate can be easily envisaged if there is a macrophase separation between the HBC and PDI molecules. Note
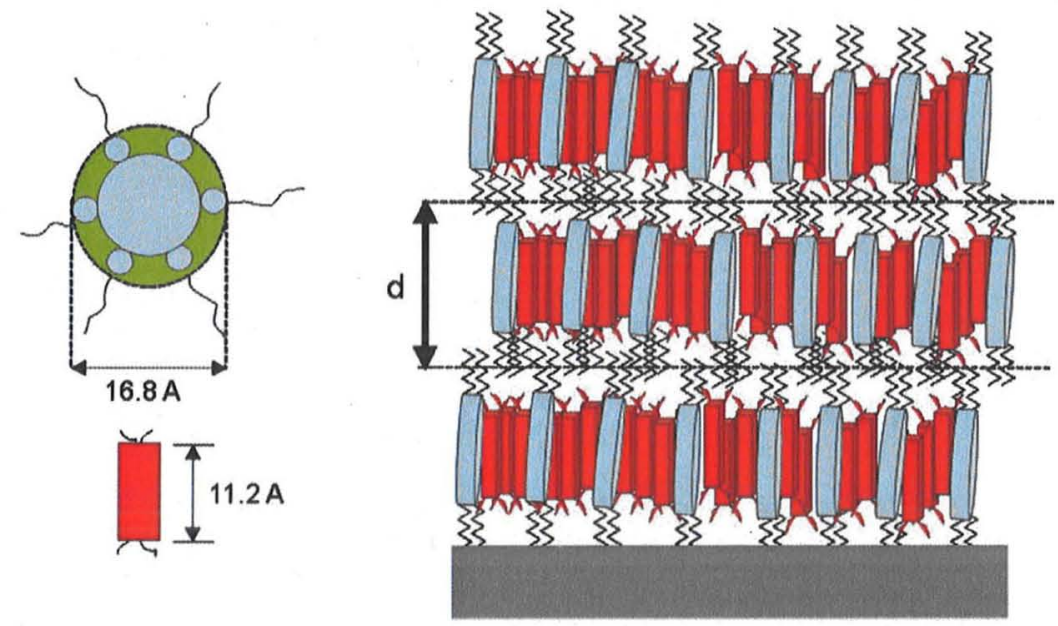

Fig. 8. Schematic drawing for a possible model of the structure of HBC:PDI blend films. 
that the effective interaction parameter between the alkyl side chains and the aromatic cores of the $\mathrm{HBC}$ molecules is the highest in this blend, nonetheless it cannot induce a macrophase separation between the HBC and PDI molecules. This entails unfavorable contact between the aromatic parts and the alkyl side chains (large enthalpic penalty), and it must be compensated from another interaction in the system. Apparently, the gain in free energy due to the formation of donoracceptor complexes overweighs this enthalpic penalty of mixing for blends with long side chains $\mathrm{HBC}$ molecules. This is in line with the absence of experimental indications of a layered structure. These findings highlight the importance of the donor-acceptor interaction in one hand, and the size difference between their conjugated parts on the other in determining the structure of the active layer.

The results presented thus far provide some clues to understanding the observed increase of photovoltaic device performance for the blends with short side chain HBC molecules for which higher charge carrier generation yield, and higher power conversion efficiencies are found [1]. As we discussed above, the formation of donor-acceptor complexes seems to be formed in all blends. Blends with short side chain HBC molecules allow for packing of these complexes in a layered structure and in turn large domains are formed. In contrast, for blends with long side chain HBC molecules, the structure is almost disordered with order presents on a local scale only. Such disordered structure may explain the radiative and non-radiative loss mechanisms of excited molecules, and consequently lower charge carrier mobility (lower currents) observed for these materials as shown by photocurrent decay (PCD) and photoluminescence (PL) measurements in our previous work [1]. Although, the blends with short side chain HBC molecules show higher power conversion efficiencies, their values are considered low when compared with values reported for other systems such as conjugated polymer $/ \mathrm{C}_{60} \mathrm{BHJ}$ solar cells [40]. This can be ascribed to two effects. First, the morphology of the HBC-6:PDI and $\mathrm{HBC}-8$ :PDI is not ideal. The intimate contact between the HBC and PDI molecules in each layer leads to more charge separation and thus high PL quenching. On the other hand, the charges generated at the interfaces do not have direct pathways to the electrodes and therefore cannot exit the device easily. This results in strong bimolecular recombination [39]. Second, our results indicate that a large fraction of the intermixed donor-acceptor layers are comprised of edge-on molecules (parallel layers to the substrate). This is not a favorable alignment as it leads to poor charge mobility perpendicular to the electrodes. Because of the large separation of the conjugated layers, lateral charge carrier hopping between adjacent layers is necessary for current generation. In contrast, face-on aligned molecules would facilitate faster charge transport towards the electrodes leading to improved charge extraction [41]. This explains the low fill factor, FF, high shunt resistance and significant deviation of short-circuit current, $I_{S C}$ under solar light compared to the high $I_{S C}$ calculated from low intensity EQE data for the devices of blends with short side chain HBCs [1]. These two effects must be considered for the optimization of devices based on discotic materials. Systems comprised of small donor-acceptor molecules that phase separate and give higher power conversion efficiencies have been reported in the literature $[42,43]$. It is anticipated that the combined control of morphology, miscibility, and alignment of the active layer of these systems would pave the way to efficient solar cells and other electronic devices.

\section{Conclusions}

We have studied the surface morphology and inner structure of blend thin films comprised of different phenyl-substituted hexa-perihexabenzocoronene $(\mathrm{HBC})$ molecules with varying alkyl side chain length, $\mathrm{n}=6,8,12$ and 16 carbon atoms, blended with rod-shaped perylenediimide (PDI). These blends were used as active layers in bulk-hetero junction organic solar cells we studied recently and therefore this study aims to understand the photovoltaic performance of these cells.

Scanning electron microscopy images of the surface topology of the blends reveal flake-like domains for the HBC-6:PDI, and HBC-8: PDI blends. The domains become smaller for the HBC-12:PDI blend, whereas the HBC16-PDI blend shows a rather fine texture. The inner structure of pure and blend films were studied by X-ray diffraction measurements. The results indicate that microphase separation of the aliphatic and aromatic parts of both pure $\mathrm{HBC}$ and PDI molecules takes place and leads to a layered structure parallel to the substrate. Indications of sharp interfaces and a very regular repetition of these layers over a long distance of correlation length were observed for the $\mathrm{HBC}$ $12, \mathrm{HBC}-16$ and PDI pure films. In contrast to the pure HBC layers, the blends develop a layered structure that is insensitive to the side chain length, at least up to $n=12$. The layered microstructure becomes more disordered with increasing the side chain length of the HBC molecules, and eventually a predominantly disordered structure is formed in the HBC-16:PDI blend.

We propose that the presence of donor-acceptor interaction stabilizes the intercalation of PDI (the acceptor) within layers of $\mathrm{HBC}$ disks leading to a stacking morphology. The size difference between the two aromatic parts in the HBC and PDI molecules results in a room for the side chains of the two molecules to fill in the space around the aromatic cores. For $\mathrm{n}=12$, the side chains start to disrupt this layered structure. For side chain with $n>12$, the empty space produced by the size difference will not be enough to accommodate this increase leading to the disruption of the layered structure and instead a rather disordered structure is formed. Short alkyl side chains of the HBC molecules allow for packing of these complexes into an ordered layered structure and in turn large domains are formed. In contrast, for longer side chain $\mathrm{HBCs}$, the structure is almost disordered with order present on a local scale only. Such disordered structure may explain the radiative and non-radiative loss mechanisms of excited molecules, and consequently lower charge carrier mobility (lower currents) observed for these materials in our previous work. Our results indicate that a large fraction of the layers of the intermixed donor-acceptor phase for short chain HBCs is comprised of edge-on molecules (layers parallel to the substrate). This is not a favorable alignment as it leads to poor charge mobility perpendicular to the electrodes. In contrast, faceon aligned molecules would facilitate faster charge transport towards the electrodes leading to improved charge extraction.

\section{Acknowledgment}

M.A.H. thanks the German research foundation (DFG) for supporting a summer visit. This work was financially supported by DFG in the program SPP1355: elementary processes of organic photovoltaics and the program of the Cluster of Excellence. Also, we would like to acknowledge the Nano Initiative Munich (NIM).We would like to thank the groups of Professors Feldmann, Raedler and Kotthaus at the LMU for giving us access to their equipment. M.A.H. thanks Bert Nickel and Martin Huth for their assistance in performing the X-ray diffraction measurements and many fruitful discussions.

\section{References}

[1] H.C. Hesse, J. Weickert, M. Al-Hussein, L. Dössel, X. Feng, K. Müllen, L. SchmidtMende, Sol. Energy Mater. Sol. Cells 94 (2010) 560.

[2] C.J. Brabec, V. Dyakonov, J. Parisis, N.S. Sariciftci (Eds.), Organic Photovoltaic: Concepts and Realization, Springer, Berlin, 2003.

[3] G.L.V. Shrotriya, J.S. Huang, Y. Yao, T. Moriarty, K. Emery, Y. Yang, Nat. Mater. 4 (2005) 864

(4] P.E. Keivanidis, I.A. Howard, R.H. Friend, Adv. Func. Mater. 18 (2008) 3189.

[5] S.H. Park, A. Roy, S. Beaupre, S. Cho, N. Coates, J.S. Moon, D. Moses, M. Leclerc, K. Lee, A.J. Heeger, Nat Photon 3 (2009) 297.

[6] J. Roncali, Acc. Chem. Res. 42 (2009) 1719.
[7] D. Adam, P. Schuhmacher, J. Simmerer, L. Haussling, K. Siemensmeyer, K.H Etzbach, H. Ringsdorf, D. Haarer, Nature 371 (1994) 141. 
[8] J. Simmerer, B. Glusen, W. Paulus, A. Kettner, P. Schuhmacher, D. Adam, K.H. Etzbach, K. Siemensmeyer, J.H. Wendorff, H. Ringsdorf, D. Haarer, Adv. Mater. 8 (1996) 815.

[9] H. Iino, Y. Takayashiki, J. Hanna, R.J. Bushby, D. Haarer, Appl. Phys. Lett. 87 (2005) 192105.

[10] I. Fischbach, T. Pakula, P. Minkin, A. Fechtenkötter, K. Müllen, H.W. Spiess, K. Saalwächter, J. Phys. Chem. B 106 (2002) 6418.

[11] L. Schmidt-Mende, A. Fechtenkötter, K. Müllen, E. Moons, R.H. Friend, J.D. MacKenzie, Science 293 (2001) 1119.

[12] A.M. van de Craats, J.M. Warman, Adv. Mater. 13 (2001) 130

[13] S.K. Sadhukhan, C. Viala, A. Gourdon, Synthesis 10 (2003) 1521.

[14] R. Rathore, C.L. Burns, J. Org. Chem. 68 (2003) 4071.

[15] S. Sergeyev, W. Pisula, Y.H. Geerts, Chem. Soc. Rev. 36 (2007) 1902.

[16] A.M. van de Craats, J.M. Warman, A. Fechtenkötter, J.D. Brand, M.A. Harbison, K. Müllen, Adv. Mater. 11 (1999) 1469.

[17] J.M. Warman, M.P. de Haas, G. Dicker, F.C. Grozema, J. Piris, M.G. Debije, Chem. Mater. 16 (2004) 4600.

[18] W. Pisula, A. Menon, M. Stepputat, I. Lieberwirth, U. Kolb, A. Tracz, H. Sirringhaus, T. Pakula, K. Müllen, Adv. Mater. 17 (2005) 684.

[19] P. Miskiewicz, A. Rybak, J. Jung, I. Glowacki, J. Ulanski, Y.H. Geerts, M. Watson, K. Müllen, Synth. Met. 137 (2003) 905.

[20] P. Herwig, C. Kayser, K. Müllen, H.W. Spiess, Adv. Mater. 8 (1996) 510.

21] M. Watson, A. Fechtenkötter, K. Müllen, Chem. Rev. 101 (2001) 1267.

[22] P. Samori, A. Fechtenkötter, F. Jäckel, T. Böhme, K. Müllen, J.P. Rabe, J. Am. Chem. Soc, 123 (2001) 462

[23] M. Lee, J.-W. Kim, S. Peleshanko, K. Larson, Y.S. Yoo, D. Vaknin, S. Markutsya, V.V. Tsukruk, J. Am. Chem. Soc. 124 (2002) 9121

[24] A. Fechtenkötter, K. Saalwaechter, M. Harbison, K. Müllen, H.W. Spiess, Angew. Chem. Int. Ed. 38 (1999) 3039.

[25] C. Waldauf, M. Morana, P. Denk, P. Schilinsky, K. Coakley, S.A. Choulis, C.J. Brabec, Appl. Phys. Lett. 89 (2006) 233517-1-3
[26] R. Steim, S.A. Choulis, P. Schilinsky, C.J. Brabec, Appl. Phys. Lett. 92 (2008) $093303-$ $1-3$

[27] G.K. Mor, K. Shankar, M. Paulose, O.K. Varghese, C.A. Grimes, Appl. Phys. Lett. 91 (2007) 152111-1-3.

[28] K. Takahashi, K. Seto, T. Yamaguchi, Ji. Nakamura, C. Yokoe, K. Murata, Chem. Lett. 33 (2004) 1042.

[29] K. Takahashi, T. Nakanishi, T. Yamaguchi, J.-I. Nakamura, K. Murata, Chem. Lett. 34 (2005) 714.

[30] J.P. Schmidtke, R.H. Friend, M. Kastler, K. Müllen, J. Chem. Phys. 124 (2006) $174704-1$.

[31] B.D. Cullity, Elements of X-Ray Diffraction, Addison-Wesley, Reading, MA, 1956

[32] D.G. Neerinck, T.J. Vink, Thin Solid Films 278 (1996) 12.

[33] D. Dambournet, I. Belharouak, K. Amine, Chem. Mater. 22 (2010) 1173.

[34] I.K. Robinson, G.S. Brown, D.E. Moncton (Eds.), Handbook on Synchrotron Radiation, vol. 3, Elsevier Science, Amsterdam, 1991

[35] H.J. Fell, E.J. Samuelsen, J. Als-Nielsen, G. Grübel, J. Mädalen, Solid State Commun. 94 (1995) 843.

[36] H. Dosch, B.W. Batterman, D.C. Wack, Phys, Rev, Lett. 56 (1986) 1144

[37] G. Zucchi, B. Donnio, Y.H. Geerts, Chem. Mater. 17 (2005) 4274

38] G. Zucchi, P. Viville, B. Donnio, A. Vlad, S. Melinte, M. Mondeshki, R. Graf, H.W. Spiess, Y.H. Geerts, R. Lazzaroni, J. Phys. Chem. B 113 (2009) 5448.

[39] D. Venkataraman, S. Yurt, H. Venkataraman, N. Gavvalapalli, Phys. Chem. Lett. 1 (2010) 947.

[40] S.E. Shaheen, C.J. Brabec, N.S. Sariciftci, Appl. Phys. Lett. 78 (2001) 841.

[41] C. Videlot, D. Fichou, Synth. Met. 102 (1999) 885

[42] C.-Q. Ma, M. Fonrodona, M.C. Schikora, M.M. Wienk, R.A.J. Janssen, P. Bäuerle, Adv. Funct. Mater. 18 (2008) 3323

[43] B. Walker, A.B. Tamayo, X.-D. Dang, P. Zalar, J.-H. Seo, A. Garcia, M. Tantiwiwat, Th.-Q. Nguyen, Adv. Funct. Mater. 19 (2009) 3063. 